\title{
Unmanipulated haploidentical in comparison with matched unrelated donor stem cell transplantation in patients 60 years and older with acute myeloid leukemia: a comparative study on behalf of the ALWP of the EBMT
}

Nicole Santoro ${ }^{1,2^{*}}$ D. Myriam Labopin ${ }^{1,3}$, Federica Giannotti ${ }^{1}$, Gerard Ehninger ${ }^{4}$, Dietger Niederwieser ${ }^{5}$, Arne Brecht ${ }^{6}$, Matthias Stelljes ${ }^{7}$, Nicolaus Kröger ${ }^{8}$, Herman Einsele ${ }^{9}$, Matthias Eder ${ }^{10}$, Michael Hallek ${ }^{11}$, Bertram Glass $^{12}$, Jürgen Finke ${ }^{13}$, Fabio Ciceri ${ }^{14}$, Mohamad Mohty ${ }^{1}$, Annalisa Ruggeri ${ }^{1,15}$ and Arnon Nagler 3,16

\begin{abstract}
Background: Acute myeloid leukemia (AML) is both more common and with more biologically aggressive phenotype in the elderly. Allogenic stem cell transplantation (allo-SCT) is the best treatment option in fit patients. Either HLAmatched unrelated donor (MUD) or haploidentical (Haplo) donor are possible alternative for patients in need.

Methods: We retrospectively compared non-T-cell-depleted Haplo $(n=250)$ to 10/10 MUD $(n=2589)$ in AML patients $\geq 60$ years.

Results: Median follow-up was 23 months. Disease status at transplant differs significantly between the two groups $\left(p<10^{-4}\right)$. Reduced intensity conditioning (RIC) was administrated to 73 and $77 \%$ of Haplo and MUD, respectively $(p=0.23)$. Stem cell source was the bone marrow (BM) in $52 \%$ of the Haplo and $6 \%$ of MUD $\left(p<10^{-4}\right)$. Anti-thymocyte globulin (ATG) was most frequently used in MUD $\left(p<10^{-4}\right)$ while post-Tx cyclophosphamide (PT-Cy) was given in $62 \%$ of Haplo. Engraftment was achieved in 90\% of the Haplo vs 97\% of MUD $\left(p<10^{-4}\right)$. In multivariate analysis, no significant difference was found between Haplo and MUD for acute (a)graft versus host disease (GVHD) grade II-IV, relapse incidence (RI), non-relapse mortality (NRM), leukemia free survival (LFS), graft-versus-host-free-relapse free survival (GRFS), and overall survival (OS). Extensive chronic (c)GVHD was significantly higher for MUD as compared to Haplo (HR 2, $p=0.01$, 95\% Cl 1.17-3.47). A propensity score analysis confirmed the higher risk of extensive CGVHD for MUD without differences for other outcomes.

Conclusions: Allo-SCT from both Haplo and MUD are valid option for AML patients $\geq 60$ years of age with similar results. Transplantation from MUD was associated with higher extensive cGVHD. Our findings suggest that Haplo is a suitable and attractive graft source for patients $\geq 60$ with AML in need of allo-SCT.
\end{abstract}

Keywords: MUD, Haploidentical, Allogeneic stem cell transplantation, Acute myeloid leukemia, Elderly

\footnotetext{
* Correspondence: nicole.santoro24@gmail.com

${ }^{1}$ Department of Hematology and Cell Therapy, Saint-Antoine Hospital, Paris,

France

${ }^{2}$ Section of Hematology, Department of Medicine, University of Perugia,

Centro Ricerche Emato-Oncologiche (CREO), Perugia, Italy

Full list of author information is available at the end of the article
} 


\section{Background}

Acute myeloid leukemia (AML) is primarily a disease of the elderly, with a median age at diagnosis of 68-72 years [1]. For these patients, prognosis still remains very poor. Recent data indicate a median survival of 3 months for patients > 65 years, with slightly higher estimates for patients aged 66-75 years (about $\sim 6$ months) than in patients 76-89 year olds (in the neighborhood of 2 . 5 months) and merely $5 \%$ of the patients are alive 5 years from diagnosis [2].

In elderly, AML is associated with poorer prognosis than in the younger, due to the frequent adverse genetic or epigenetic features present at diagnosis and the increased non-relapse mortality (NRM) [3]. Although most elderly AML patients still succumb to their disease, improvements in the prognosis have been documented in recent years, due to better supportive care, management of infections, and patient selection for chemotherapy based on comorbidity scores [4]. Allogenic stem cell transplantation (allo-SCT) remains the most effective anti-leukemic treatment in AML, but the toxicity of the procedure and the difficulty to allocate a suitable donor have limited allo-SCT to a small fraction of patients in need [5]. Both the development of reduced conditioning regimens (RIC) [6-8] and the use of alternative donors improved the accessibility to transplant for high-risk patients in need. Indeed, a recent study reports that over the past decade, utilization and survival after allo-SCT have significantly increased in patients $\geq 70$ years [9].

Availability of a donor for this high-risk patient population is still a challenge. HLA matched related donor (MRD) transplants remain the best choice for optimal transplant outcome, but only approximately $25-30 \%$ of patients have such a donor [10]. Moreover, in the elderly patients, availability of MRD is even lower, due to the increased age of family members as well as comorbidities and contraindications for stem cell donation. Other donor options include HLA-matched unrelated donors (MUD), HLA-mismatched unrelated donors 9/10 (MMUD), umbilical cord blood units (UCB), and haploidentical family donors. MUD or MMUD are frequently used when a suitable MRD is lacking, with similar transplantation outcomes [11-14]. However, these reports focus mainly on young patients receiving myeloablative conditioning (MAC), and there are only few data from older populations [15]. In the absence of MRD, MUD is one of the possible choices but the probability to find a donor is estimated to be around 75\% in Caucasians, 46\% in Middle East or North African origin, and only $16 \%$ in African-Americans [16]. In addition, the search may take few weeks or even months while waiting for a volunteer donor; the leukemia may reoccur.

Over the last recent years, haploidentical donors have been increasingly used as a valid alternative for allo-SCT
[17]. Unmanipulated haploidentical transplant (Haplo-SCT) without T-cell depletion (TCD) is currently used more frequently either with anti-thymocyte globulins (ATG) or post-transplant cyclophosphamide (PT-Cy) as graft versus host disease (GVHD) prophylaxis [18-20]. The optimization of conditioning regimens has further extended the use of Haplo-SCT to older patients and those with significant pre-transplant comorbidities. However, while the feasibility of allo-SCT from an MRD using reduced intensity conditioning (RIC) regimens in elderly patients was demonstrated in several studies [21-23], only few studies reported the outcomes of Haplo-SCT in elderly patients mainly using RIC and PT-Cy-based GVHD prophylaxis [24-29].

With the aim to better define the role of alternative donors in elderly patients with AML, we performed a large registry-based study, with the European Society of Bone Marrow Transplantation (EBMT)-Acute Leukemia Working Party (ALWP), comparing non-TCD HaploSCT to transplants from MUD for AML patients 60 years and older.

\section{Methods \\ Study design and definition}

This is a retrospective registry-based analysis on behalf of the ALWP of EBMT. The EBMT is a non-profit, scientific society representing more than 600 transplant centers mainly in Europe that are required to report all consecutive stem cell transplantations and follow-ups once a year. Data are entered, managed, and maintained in a central database with internet access; each EBMT center is represented in this database. Audits are routinely performed to determine the accuracy of the data. Patients provide informed consent authorizing the use of their personal information for research purposes.

Eligibility criteria included all adults $\geq 60$ years with AML, who underwent a first allo-SCT between January 2007 and December 2014, using either a 10/10 MUD or Haplo from a family donor (recipient-donor number of mismatches $\geq 2$ ). Transplants were performed in 210 EBMT centers.

MAC was defined as a regimen containing either total body irradiation (TBI) with a dose greater than $6 \mathrm{~Gy}$, a total dose of oral busulfan $(\mathrm{Bu})$ greater than $8 \mathrm{mg} / \mathrm{kg}$, or a total dose of intravenous $\mathrm{Bu}$ greater than $6.4 \mathrm{mg} / \mathrm{kg}$ or melphalan at doses $>140 \mathrm{mg} / \mathrm{m}^{2}$. In addition, regimens containing two alkylating agents were considered as MAC. All other regimens were defined as RIC [30]. Cytogenetics abnormalities were classified according to the 2010 European Leukemia Net cytogenetic classification system [31].

\section{Endpoints}

The primary endpoint was leukemia-free survival (LFS). Secondary endpoints were overall survival (OS), refined 
graft-versus-host-free, relapse-free survival (GRFS), neutrophil engraftment, acute (a)GVHD and chronic (c) GVHD, relapse incidence (RI), and non-relapse mortality (NRM). LFS was defined as the interval from HaploSCT to either relapse or death in remission. OS was defined as the time to death from all causes. GRFS events have been defined as grade III-IV aGVHD, severe cGVHD, disease relapse, or death from any cause after SCT [32]. Engraftment was defined as the first of three consecutive days with an absolute neutrophil count $>0$. $5 \times 10^{9} / \mathrm{l}$. aGVHD was graded according to the modified Glucksberg criteria [33] and cGVHD according to the revised Seattle criteria [34].

\section{Statistical analysis}

Patient-, disease-, and transplant-related variables were compared between the two groups (Haplo or MUD) using the chi-square statistic for categorical variables and the Mann-Whitney test for continuous variables. Factors that differ significantly between the two groups with $p$ values of $<0.05$ and all factors known as potential prognostic factors were included in the final models. Cumulative incidence (CI) of relapse and NRM was calculated from the date of transplant to the date of relapse or death in remission, respectively, with the other event being the competing risk. For studying GVHD, both relapse and death were considered as competing events. Univariate comparisons of time-dependent endpoints were done using the log-rank test for OS and LFS and GRFS and the Gray's test for cumulative incidence functions. A multivariate analysis was performed using Cox proportional hazards model. Variables were included in the multivariate model if they were conceptually important or if they approached or attained statistical significance by univariate analysis. All tests are two-sided. The type I error rate was fixed at 0.05 for determination of factors associated with time to event. In order to test for a center effect, we introduced a random effect or frailty for each center into the model. Statistical analyses were performed with the SPSS 22 (SPSS Inc./IBM, Armonk, NY, USA) and R 3.2.3 (R Development Core Team, Vienna, Austria) software package.

To allow for potential confounding factors between treatments that could influence outcome, propensity score matching was also performed [35]. The following factors were included in the propensity score model: patient age, year of transplant, cytogenetics, status at transplant (Tx), sex, female donor to male recipient vs other combinations, Karnofsky performance status less or more than $90 \%$, patient and donor CMV serology, stem cell source (peripheral blood stem cell (PBSC) vs BM), use of in vivo T-cell depletion, conditioning (MAC vs RIC), and previous autograft. Owing to the significant differences in baseline characteristics between the haplo and MUD groups, caliper matching was fixed to 0.2. The purpose of the propensity score matching strategy was to reduce confounding effects of these variables and strengthen causal inferences. Propensity score analysis was performed using the "MatchIt" (https://cran.r-project.org/web/packages/MatchIt/index.html). Comparisons between the two match-paired groups were stratified on matching group.

\section{Results}

Patients, disease, and transplant characteristics

We analyzed 250 AML patients $\geq 60$ years, receiving Haplo-SCT and 2589 patients transplanted from MUD between 2007 and 2014. Patients and transplant characteristics are summarized in Table 1.

Median follow-up was 23 months for both Haplo and MUD. Haplo-SCT were performed more recently, median year of transplant 2013 vs 2012 for MUD, respectively $\left(p<10^{-4}\right)$. Median time from diagnosis to transplant was longer for Haplo (9 vs 6.8 months, respectively, $p=0.001$ ). The majority of patients had a Karnofsky performance status (KPS) $\geq 90 \%$, being $59 \%$ for Haplo and $70 \%$ for MUD, $p=0.001$. The median age was 65 years (range 60-78) for both groups. Disease status was significantly different between the two groups $\left(p<10^{-4}\right)$. Secondary AML was diagnosed in $37 \%$ of haplo and 35\% of MUD. As for cytogenetics, it was intermediate risk in 17\% of Haplo and 15\% of MUD, while 18 and 16\% of the Haplo and MUD harbor poorrisk cytogenetics, respectively $(p=0.03)$.

Stem cell source, GVHD prophylaxis, and conditioning regimens are provided in Table 2.

Stem cell source was the bone marrow (BM) in $52 \%$ of Haplo and 6\% of MUD. Most of the MUD (94\%) received PBSC grafts $\left(\mathrm{p}<10^{-4}\right)$. Cyclosporine and mycophenolate were used as the main GVHD prophylaxis in the MUD group (44.5\%) while PT-CY for GVHD prophylaxis was used in $65 \%$ of Haplo. Finally, T-cell depletion with ATG significantly differs between the two groups ( 74 vs $26 \%$, respectively, $p<10^{-4}$ ) (Table 2). In order to overcome these differences, the propensity score technique was applied and we were able to match 225 haplo with 450 MUD using propensity score matching.

Engraftment and acute and chronic graft versus host disease The 60 days CI of neutrophil engraftment was $90 \%$ for the Haplo and $97 \%$ for the MUD $\left(p<10^{-4}\right)$.

In the multivariate analysis (Table 3), the risk of grade II-IV aGVHD (HR 1.17, $p=0.37,95 \%$ CI $0.82-1.65$ ) and cGVHD (HR1.21, $p=0.28$, 95\% CI 0.84-1.75) was not associated to the type of donor. However, recipients of MUD experienced higher risk of extensive cGVHD compared to Haplo (HR 2, $p=0.01,95 \%$ CI 1.17-3.47). 
Table 1 Patients and donors characteristics

\begin{tabular}{|c|c|c|c|}
\hline & Haplo & MUD 10/10 & $p$ value \\
\hline Number & 250 & 2589 & \\
\hline Follow-up & 23.18 (1.8-93.9) & $23.02(0.2-128)$ & \\
\hline Year of Tx & 2013 (2006-2014) & 2012(2001-2014) & $<10^{-4}$ \\
\hline Time to Tx (months) & $9(5.1-18.7)$ & $6.8(4.6-15)$ & 0.001 \\
\hline \multicolumn{4}{|l|}{ Patient sex } \\
\hline Male & $156(62 \%)$ & 1471 (57\%) & \multirow[t]{3}{*}{0.093} \\
\hline Female & $94(38 \%)$ & $1114(43 \%)$ & \\
\hline Missing & 0 & 4 & \\
\hline \multicolumn{4}{|l|}{ Performance status } \\
\hline KPS $\leq 90$ & $97(41 \%)$ & 723 (28\%) & \multirow[t]{3}{*}{0.001} \\
\hline KPS $\geq 90$ & 139 (59\%) & $1670(65 \%)$ & \\
\hline Missing & $14(0 \%)$ & $196(7 \%)$ & \\
\hline Age & $65(62.3-66.9)$ & $64.8(62.2-67.6)$ & 0.756 \\
\hline \multicolumn{4}{|l|}{ Disease status } \\
\hline CR1 & $95(38 \%)$ & 1377 (53\%) & \multirow[t]{4}{*}{$<10^{-4}$} \\
\hline$C R \geq 2$ & 46 (18\%) & $436(17 \%)$ & \\
\hline Active disease & 109 (44\%) & 776 (30\%) & \\
\hline Missing & 0 & 0 & \\
\hline \multicolumn{4}{|l|}{ Cytogenetics } \\
\hline Good & $17(10 \%)$ & $77(5 \%)$ & \multirow[t]{3}{*}{0.036} \\
\hline Intermediate & $28(17 \%)$ & $210(15 \%)$ & \\
\hline Poor & $31(18 \%)$ & $221(16 \%)$ & \\
\hline Secondary AML & $92(55 \%)$ & 902 (64\%) & 0.535 \\
\hline Missing & 82 & 1179 & \\
\hline Previous autologus Tx & $21(8 \%)$ & $49(2 \%)$ & $<10^{-4}$ \\
\hline \multicolumn{4}{|l|}{ Sex mismatch $D / R$} \\
\hline No F->M & 197 (79\%) & $2210(88 \%)$ & \multirow[t]{3}{*}{$<10^{-4}$} \\
\hline $\mathrm{F}->\mathrm{M}$ & $53(21 \%)$ & $297(12 \%)$ & \\
\hline Missing & 0 & 82 & \\
\hline
\end{tabular}

Abbreviations: Haplo haploidentical; $M U D$, matched unrelated donor; $T X$, transplant; $K P S$, Karnofsky performance status; $C R$, complete remission; $A M L$, acute myeloid leukemia; $F$, female; $M$, male; $D$, donor; $R$, recipient

In vivo $\mathrm{T}$-cell depletion was associated with a reduced risk of aGVHD (HR 0.699, 95\% CI $0.57-0.858, p<0.01$ ) and cGVHD (HR 0.57, 95\% CI 0.4-0.7, $p<0.01$ ) while the use of PBSC as stem cell source increased risk of cGVHD (HR 1.48, 95\% CI 1.08-2.03, $p=0.01$ ).

These findings were confirmed in the pair-matched analysis, with a significantly increased risk of extensive cGVHD (20.5 vs $10.7 \%, p=0.0041$ ) in MUD vs Haplo (Fig. 1).

\section{Relapse incidence and NRM}

In multivariate analysis (Table 3), there was no difference in RI according to the type of donor. Factors independently associated with increased risk of relapse were disease status at transplantation (HR 1.96, 95\%
Table 2 Stem cell source, GVHD prophylaxis, and conditioning regimens

\begin{tabular}{|c|c|c|c|}
\hline & $\begin{array}{l}\text { Haplo, } \\
n(\%)\end{array}$ & $\begin{array}{l}\text { MUD 10/10, } \\
n(\%)\end{array}$ & $p$ value \\
\hline \multicolumn{4}{|l|}{ Stem cell source } \\
\hline BM & $129(52)$ & $167(6)$ & \multirow[t]{3}{*}{$<10^{-4}$} \\
\hline PBSC & $121(48)$ & $2422(94)$ & \\
\hline Missing & 0 & 0 & \\
\hline \multicolumn{4}{|l|}{ GVHD prophylaxis } \\
\hline Csa based & $4(2)$ & $426(17)$ & \multirow[t]{9}{*}{$<10^{-4}$} \\
\hline Csa + Mtx & $9(4)$ & $656(26)$ & \\
\hline Csa $+\mathrm{Mmf} \pm$ others & $12(5)$ & $1123(44)$ & \\
\hline$C S A+M M F \pm M T X$ & $16(7)$ & $29(1)$ & \\
\hline Tacro alone & $1(0)$ & $20(1)$ & \\
\hline Mmf + Tacro/Mmf + Siro & $38(16)$ & $185(7)$ & \\
\hline Mtx + Tacro & $0(0)$ & $44(2)$ & \\
\hline Pt-Cy based & $156(65)$ & $22(1)$ & \\
\hline Others & $14(1)$ & $84(1)$ & \\
\hline \multicolumn{4}{|l|}{ In vivo TCD } \\
\hline No & $181(74)$ & $648(25)$ & \multirow[t]{3}{*}{$<10^{-4}$} \\
\hline Yes & $63(26)$ & $1915(75)$ & \\
\hline Missing & 6 & 26 & \\
\hline \multicolumn{4}{|l|}{ Conditioning regimens } \\
\hline MAC & $66(27)$ & $591(23)$ & \multirow[t]{12}{*}{0.238} \\
\hline Bu-Cy/BuFlu & 7 & 253 & \\
\hline TBF & 31 & 8 & \\
\hline Flu-Mel & 7 & 81 & \\
\hline TBI based & 4 & 61 & \\
\hline Other & 17 & 188 & \\
\hline $\mathrm{RIC}$ & $182(73)$ & $1948(77)$ & \\
\hline Bu-Flu & 10 & 635 & \\
\hline TBF & 55 & 19 & \\
\hline Flu-Mel & 9 & 414 & \\
\hline TBI based & 55 & 457 & \\
\hline Other & 53 & 423 & \\
\hline
\end{tabular}

Abbrevations: Haplo, haploidentical; $M U D$, matched unrelated donor; $B M$, bone marrow; PBSC, peripheral blood stem cell; GVHD, graft versus host disease; CSA, cyclosporine; $M T X$, methotrexate; $M M F$, mycophenolate; TACRO, tacrolimus; SIRO, sirolimus; $P T C Y$, post-transplant cyclophosphamide; TCD, T-cell depletion; MAC, myeloablative conditioning; $B U$, busulphan, $C Y$, cyclophosphamide; $F L U$, fludarabine; $T B F$, thiotepa busulphan fludarabine; MEL, melphalan; TREO, treosulphan; FLAMSA, fludarabine, amsacrine, and cytarabine; $T B I$, total body irradiation; $R I C$, reduced intensity conditioning

CI $1.65-2.34, p<0.01$ ) and cytogenetics risk (poor vs good) $(p=0.002)$ (Table 3$)$.

The most common causes of death were disease recurrence (35\% in the haplo and $45 \%$ in the MUD group, respectively), infections ( 35 vs $26 \%$ ), and GVHD (18 vs $16 \%$ ).

In multivariate analysis, the type of donor did not influence the risk of NRM. Increased age (HR 1.342, $p=0.003$, 
Table 3 Multivariate analysis

\begin{tabular}{|c|c|c|c|}
\hline & $H R$ & $\mathrm{Cl}$ & $p$ \\
\hline \multicolumn{4}{|l|}{ LFS } \\
\hline MUD vs Haplo & 0.94 & $0.76-1.17$ & 0.630 \\
\hline Year of Tx & 1.01 & $0.98-1.03$ & 0.445 \\
\hline Age (per 10 years) & 1.10 & $0.95-1.27$ & 0.173 \\
\hline \multicolumn{4}{|l|}{ Status at Tx } \\
\hline$C R \geq 2$ vs $C R 1$ & 1.22 & $1.05-1.42$ & 0.009 \\
\hline Advanced vs CR1 & 1.67 & $1.48-1.89$ & $<10^{-4}$ \\
\hline \multicolumn{4}{|l|}{ Cytogenetics } \\
\hline Intermediate vs good & 1.10 & $0.76-1.60$ & 0.590 \\
\hline Poor vs good & 1.79 & $1.25-2.56$ & 0.001 \\
\hline Secondary vs good & 1.49 & $1.06-2.08$ & 0.019 \\
\hline Missing vs good & 1.40 & $1.01-1.95$ & 0.043 \\
\hline Female $\mathrm{D} /$ male $\mathrm{R}$ & 1.09 & $0.93-1.27$ & 0.247 \\
\hline KPS $\geq 90 \%$ & 0.85 & $0.76-0.96$ & 0.009 \\
\hline PBSC vs BM & 1.04 & $0.86-1.25$ & 0.670 \\
\hline In vivo TCD & 0.93 & $0.82-1.05$ & 0.288 \\
\hline Previous autograft & 1.36 & $1.00-1.86$ & 0.045 \\
\hline RIC vs MAC & 1.04 & $0.92-1.18$ & 0.468 \\
\hline Center (frailty) & & & 0.292 \\
\hline \multicolumn{4}{|l|}{ OS } \\
\hline MUD vs Haplo & 0.87 & $0.68-1.10$ & 0.244 \\
\hline Year of Tx & 1.01 & $0.98-1.04$ & 0.392 \\
\hline Age (per 10 years) & 1.16 & $1.00-1.34$ & 0.046 \\
\hline \multicolumn{4}{|l|}{ Status at Tx } \\
\hline$C R \geq 2$ vs $C R 1$ & 1.16 & $0.99-1.37$ & 0.059 \\
\hline Advanced vs CR1 & 1.62 & $1.42-1.85$ & $<10^{-4}$ \\
\hline \multicolumn{4}{|l|}{ Cytogenetics } \\
\hline Intermediate vs good & 1.05 & $0.71-1.54$ & 0.792 \\
\hline Poor vs good & 1.66 & $1.14-2.40$ & 0.007 \\
\hline Secondary vs good & 1.40 & $0.99-1.98$ & 0.057 \\
\hline Missing vs good & 1.32 & $0.94-1.87$ & 0.105 \\
\hline Female D/male R & 1.14 & $0.97-1.34$ & 0.093 \\
\hline KPS $\geq 90 \%$ & 0.83 & $0.74-0.94$ & 0.003 \\
\hline PBSC vs BM & 1.01 & $0.83-1.24$ & 0.893 \\
\hline in vivo TCD & 0.93 & $0.81-1.08$ & 0.372 \\
\hline Previous autograft & 1.39 & $1.00-1.93$ & 0.043 \\
\hline RIC vs MAC & 1.12 & $0.97-1.28$ & 0.096 \\
\hline Center (frailty) & & & 0.062 \\
\hline \multicolumn{4}{|l|}{$\mathrm{Rl}$} \\
\hline MUD vs Haplo & 1.06 & $0.76-1.47$ & 0.699 \\
\hline Year of Tx & 0.99 & $0.96-1.03$ & 0.790 \\
\hline Age (per 10 years) & 0.87 & $0.71-1.08$ & 0.230 \\
\hline \multicolumn{4}{|l|}{ Status at Tx } \\
\hline$C R \geq 2$ vs $C R 1$ & 1.26 & $1.01-1.57$ & 0.033 \\
\hline
\end{tabular}

Table 3 Multivariate analysis (Continued)

\begin{tabular}{|c|c|c|c|}
\hline & $H R$ & $\mathrm{Cl}$ & $p$ \\
\hline Advanced vs CR1 & 1.96 & $1.65-2.34$ & $<10^{-4}$ \\
\hline \multicolumn{4}{|l|}{ Cytogenetics } \\
\hline Intermediate vs good & 0.95 & $0.57-1.60$ & 0.869 \\
\hline Poor vs good & 2.11 & $1.29-3.43$ & 0.002 \\
\hline Secondary vs good & 1.35 & $0.85-2.14$ & 0.202 \\
\hline Missing vs good & 1.31 & $0.83-2.06$ & 0.241 \\
\hline Female $\mathrm{D} /$ male $\mathrm{R}$ & 0.88 & $0.69-1.11$ & 0.285 \\
\hline KPS $\geq 90 \%$ & 0.96 & $0.82-1.14$ & 0.711 \\
\hline PBSC vs BM & 1.02 & $0.77-1.34$ & 0.865 \\
\hline in vivo TCD & 1.04 & $0.86-1.25$ & 0.662 \\
\hline Previous autograft & 1.16 & $0.71-1.88$ & 0.546 \\
\hline RIC vs MAC & 0.91 & $0.76-1.10$ & 0.374 \\
\hline Center (frailty) & & & 0.135 \\
\hline \multicolumn{4}{|l|}{ NRM } \\
\hline MUD vs Haplo & 0.75 & $0.54-1.05$ & 0.095 \\
\hline Year of Tx & 1.02 & $0.98-1.06$ & 0.243 \\
\hline Age (per 10 years) & 1.34 & $1.10-1.63$ & 0.003 \\
\hline \multicolumn{4}{|l|}{ Status at Tx } \\
\hline$C R \geq 2$ vs $C R 1$ & 1.18 & $0.94-1.47$ & 0.135 \\
\hline Advanced vs CR1 & 1.45 & $1.21-1.74$ & $<10^{-4}$ \\
\hline \multicolumn{4}{|l|}{ Cytogenetics } \\
\hline Intermediate vs good & 1.29 & $0.75-2.22$ & 0.353 \\
\hline Poor vs good & 1.46 & $0.84-2.51$ & 0.172 \\
\hline Secondary vs good & 1.63 & $0.99-2.68$ & 0.051 \\
\hline Missing vs good & 1.49 & $0.91-2.43$ & 0.111 \\
\hline Female $\mathrm{D} /$ male $\mathrm{R}$ & 1.34 & $1.08-1.65$ & 0.005 \\
\hline KPS $\geq 90 \%$ & 0.75 & $0.63-0.89$ & 0.001 \\
\hline PBSC vs BM & 1.08 & $0.82-1.43$ & 0.564 \\
\hline In vivo TCD & 0.85 & $0.70-1.04$ & 0.122 \\
\hline Previous autograft & 1.62 & $1.07-2.46$ & 0.022 \\
\hline RIC vs MAC & 1.19 & $0.98-1.43$ & 0.066 \\
\hline Center (frailty) & & & 0.015 \\
\hline \multicolumn{4}{|l|}{ GRFS } \\
\hline MUD vs Haplo & 1.18 & $0.95-1.47$ & 0.125 \\
\hline Year of Tx & 0.99 & $0.97-1.02$ & 0.792 \\
\hline Age (per 10 years) & 1.03 & $0.90-1.18$ & 0.606 \\
\hline \multicolumn{4}{|l|}{ Status at Tx } \\
\hline$C R \geq 2$ vs $C R 1$ & 1.16 & $1.00-1.34$ & 0.038 \\
\hline Advanced vs CR1 & 1.64 & $1.45-1.84$ & $<10^{-4}$ \\
\hline \multicolumn{4}{|l|}{ Cytogenetics } \\
\hline Intermediate vs good & 1.24 & $0.87-1.75$ & 0.223 \\
\hline Poor vs good & 1.69 & $1.20-2.38$ & 0.002 \\
\hline Secondary vs good & 1.52 & $1.11-2.09$ & 0.008 \\
\hline Missing vs good & 1.45 & $1.06-1.98$ & 0.019 \\
\hline
\end{tabular}


Table 3 Multivariate analysis (Continued)

\begin{tabular}{|c|c|c|c|c|c|c|c|}
\hline & $\mathrm{HR}$ & $\mathrm{Cl}$ & p & & $H R$ & $\mathrm{Cl}$ & $p$ \\
\hline Female $\mathrm{D} /$ male $\mathrm{R}$ & 1.05 & $0.90-1.21$ & 0.516 & Center (frailty) & & & 0.054 \\
\hline KPS $\geq 90 \%$ & 0.85 & $0.76-0.94$ & 0.003 & cGVHD & & & \\
\hline PBSC vs BM & 1.09 & $0.91-1.32$ & 0.313 & MUD vs Haplo & 1.22 & $0.84-1.75$ & 0.281 \\
\hline In vivo TCD & 0.75 & $0.66-0.85$ & $<10^{-4}$ & Year of Tx & 0.97 & $0.94-1.01$ & 0.228 \\
\hline Previous autograft & 1.32 & $0.97-1.80$ & 0.072 & Age (per 10 years) & 0.99 & $0.80-1.23$ & 0.964 \\
\hline RIC vs MAC & 1.00 & $0.88-1.13$ & 0.933 & Status at Tx & & & \\
\hline Center (frailty) & & & 0.093 & $C R \geq 2$ vs $C R 1$ & 1.05 & $0.84-1.30$ & 0.669 \\
\hline aGVHD II-IV & & & & Advanced vs CR1 & 1.12 & $0.92-1.37$ & 0.251 \\
\hline MUD vs Haplo & 1.17 & $0.82-1.65$ & 0.374 & Cytogenetics & & & \\
\hline Year of Tx & 1.00 & $0.96-1.04$ & 0.845 & Intermediate vs good & 1.72 & $1.01-2.91$ & 0.042 \\
\hline Age (per 10 years) & 0.89 & $0.71-1.11$ & 0.326 & Poor vs good & 1.74 & $1.01-2.99$ & 0.045 \\
\hline Status at $T x$ & & & & Secondary vs good & 1.88 & $1.14-3.10$ & 0.013 \\
\hline$C R \geq 2$ vs $C R 1$ & 1.00 & $0.79-1.27$ & 0.942 & Missing vs good & 1.66 & $1.01-2.72$ & 0.043 \\
\hline advanced vs CR1 & 1.15 & $0.95-1.40$ & 0.135 & Female $\mathrm{D} /$ male $\mathrm{R}$ & 1.02 & $0.81-1.28$ & 0.860 \\
\hline Cytogenetics & & & & KPS $\geq 90 \%$ & 1.09 & $0.91-1.31$ & 0.310 \\
\hline Intermediate vs good & 1.34 & $0.77-2.34$ & 0.296 & PBSC vs BM & 1.48 & $1.08-2.03$ & 0.013 \\
\hline Poor vs good & 1.45 & $0.83-2.53$ & 0.180 & In vivo TCD & 0.57 & $0.47-0.70$ & $<10^{-4}$ \\
\hline Secondary vs good & 1.40 & $0.84-2.34$ & 0.192 & Previous autograft & 1.64 & $0.99-2.70$ & 0.050 \\
\hline Missing vs good & 1.30 & $0.78-2.16$ & 0.303 & RIC Vs MAC & 0.82 & $0.67-1.02$ & 0.077 \\
\hline Female $\mathrm{D} /$ male $\mathrm{R}$ & 1.10 & $0.88-1.39$ & 0.378 & Center (frailty) & & & 0.001 \\
\hline$K P S \geq 90 \%$ & 0.87 & $0.73-1.05$ & 0.152 & Extensive cGVHD & & & \\
\hline PBSC vs BM & 1.01 & $0.75-1.35$ & 0.954 & MUD vs Haplo & 2.02 & $1.17-3.47$ & 0.011 \\
\hline In vivo TCD & 0.70 & $0.57-0.85$ & 0.001 & Year of Tx & 0.97 & $0.92-1.02$ & 0.298 \\
\hline Previous autograft & 2.18 & $1.42-3.36$ & $<10^{-4}$ & Age (per 10 years) & 1.05 & $0.77-1.42$ & 0.740 \\
\hline RIC vs MAC & 1.02 & $0.82-1.25$ & 0.853 & Status at Tx & & & \\
\hline Center (frailty) & & & 0.011 & $C R \geq 2$ vs $C R 1$ & 0.92 & $0.67-1.26$ & 0.618 \\
\hline aGVHD III-IV & & & & Advanced vs CR1 & 0.97 & $0.73-1.30$ & 0.874 \\
\hline MUD vs Haplo & 1.52 & $0.85-2.71$ & 0.154 & Cytogenetics & & & \\
\hline Year of Tx & 0.97 & $0.91-1.03$ & 0.369 & Intermediate vs good & 1.15 & $0.56-2.37$ & 0.693 \\
\hline Age (per 10 years) & 0.98 & $0.68-1.41$ & 0.935 & Poor vs good & 1.39 & $0.67-2.90$ & 0.371 \\
\hline Status at Tx & & & & Secondary vs good & 1.27 & $0.64-2.49$ & 0.481 \\
\hline$C R \geq 2$ vs $C R 1$ & 1.23 & $0.83-1.84$ & 0.290 & Missing vs good & 1.11 & $0.57-2.16$ & 0.751 \\
\hline Advanced vs CR1 & 1.78 & $1.31-2.43$ & $<10^{-4}$ & Female $\mathrm{D} /$ male $\mathrm{R}$ & 0.94 & $0.66-1.34$ & 0.751 \\
\hline Cytogenetics & & & & $K P S \geq 90 \%$ & 1.00 & $0.77-1.29$ & 0.997 \\
\hline Intermediate vs good & 2.27 & $0.77-6.68$ & 0.133 & PBSC vs BM & 1.30 & $0.83-2.05$ & 0.245 \\
\hline Poor vs good & 1.67 & $0.55-5.04$ & 0.361 & In vivo TCD & 0.35 & $0.27-0.46$ & $<10^{-4}$ \\
\hline Secondary vs good & 2.03 & $0.73-5.67$ & 0.173 & Previous autograft & 1.19 & $0.54-2.60$ & 0.658 \\
\hline Missing vs good & 1.69 & $0.61-4.70$ & 0.311 & RIC vs MAC & 0.74 & $0.54-1.01$ & 0.060 \\
\hline Female $\mathrm{D} /$ male $\mathrm{R}$ & 1.02 & $0.69-1.51$ & 0.895 & Center(frailty) & & & 0.022 \\
\hline$K P S \geq 90 \%$ & 0.68 & $0.52-0.91$ & 0.009 & \multirow{5}{*}{\multicolumn{4}{|c|}{$\begin{array}{l}\text { Abbreviations: LFS, leukemia-free survival; OS, overall survival; } R I \text {, relapse incidence; } \\
N R M \text {, non-relapse mortality; GRFS, graft-versus-host-free-relapse free survival; } a \text {, } \\
\text { acute; } c \text {, chronic; GVHD, graft versus host disease; } M U D \text {, matched unrelated donor; } \\
\text { Haplo, haploidentical; } T X \text {, transplant; } C R \text {, complete remission; } K P S \text {, Karnofsky } \\
\text { performance status; } P B S C \text {, peripheral blood; } B M \text {, bone marrow; } D \text {, donor; } R \text {, recipient; } \\
T C D \text {, T cell depletion; RIC, reduced intensity conditioning; } M A C \text {, } \\
\text { myeloablative conditioning }\end{array}$}} \\
\hline PBSC vs BM & 1.20 & $0.71-2.02$ & 0.478 & & & & \\
\hline In vivo TCD & 0.63 & $0.46-0.86$ & 0.004 & & & & \\
\hline Previous autograft & 2.07 & $1.03-4.15$ & 0.039 & & & & \\
\hline RIC vs MAC & 0.76 & $0.53-1.09$ & 0.147 & & & & \\
\hline
\end{tabular}

Table 3 Multivariate analysis (Continued)

Cytogenetics

Poor vs good

0.042

013

0.860

Page 6 of 10

(n) 


\section{a}

\section{CGVHD}
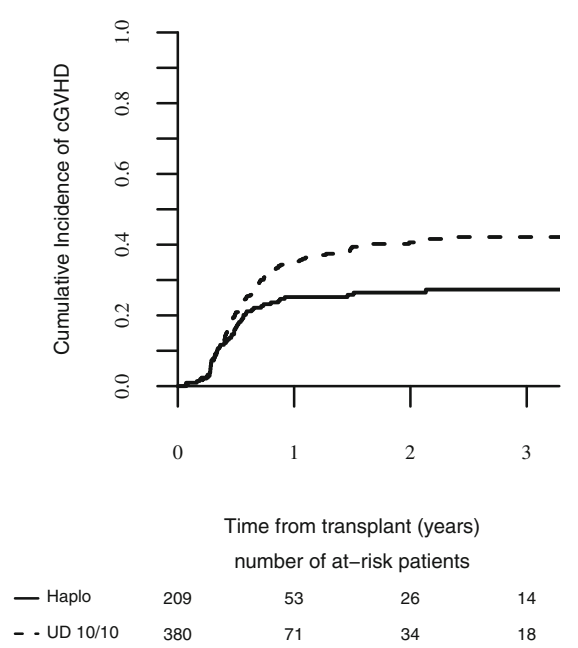

b

ext.cGVHD

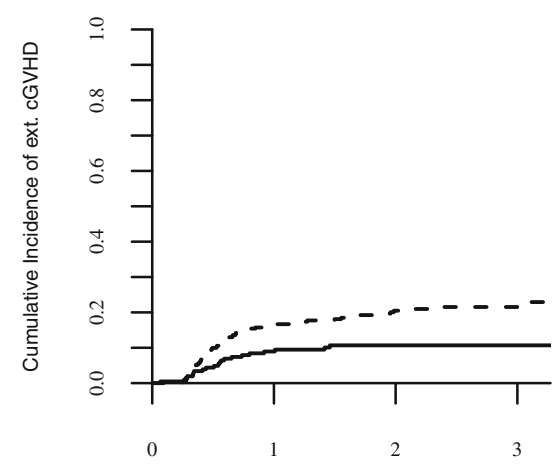

Time from transplant (years)

number of at-risk patients

$\begin{array}{lcccc}\text { - Haplo } & 209 & 76 & 39 & 23 \\ \text { - - UD 10/10 } & 380 & 119 & 66 & 35\end{array}$

Fig. 1 a) CGVHD; b) extensive CGVHD after MUD and Haplo SCT in AML patients $\geq 60$ years after matched pair analysis

95\% CI 1.102-1.634), KPS (HR 0.753, 95\% CI 0.637-0. $891, p=0.0009$ ), and advanced disease status (HR 1.454, 95\% CI 1.212-1.743, $p<10^{-4}$ ) (Table 3) were the factors independently associated with increase in mortality. These results were confirmed in the pair-matched analysis (Table 4, Fig. 2a, b).

\section{OS, LFS, and GRFS}

The median follow-up for survivors was 23 months. In adjusted multivariate analysis, OS (HR1.18, CI 0.686-1. $102, p=0.2481$ ), LFS (HR 0.94, CI 0.767-1.174, $p=0.63$ ) , and GRFS (HR1.18, CI 0.954-1.47, $p=0.125$ ) (Table 3) were comparable according to the type of donor. These results were confirmed in the pair-matched population (Table 4, Fig. 2c, d). Other independent factors influencing LFS, OS, and GRFS were disease status at transplantation (HR 1.67, 95\% CI 1.48-1.89, $p<0.01$; HR 1 . 62, 95\% CI 1.42-1.85, $p<0.01$; HR 1.64, 95\% CI 1.45-1.

Table 4 Propensity score analysis for LFS, OS, RI, NRM, GRFS, aGVHD II-IV, aGVHD III-IV, cGVHD, and ext. cGVHD

\begin{tabular}{llll}
\hline & MUD & Haplo & $p$ value \\
\hline LFS & $39.9 \%[34.7-45.1]$ & $34.6 \%[27.9-41.3]$ & 0.67 \\
OS & $42 \%[36.7-47.4]$ & $38.7 \%[31.8-45.5]$ & 0.33 \\
RI & $31.9 \%[27.2-36.7]$ & $27.7 \%[21.7-34]$ & 0.17 \\
NRM & $28.2 \%[23.6-32.9]$ & $37.7 \%[31.1-44.3]$ & 0.06 \\
GRFS & $24.8 \%[20.3-29.4]$ & $30.2 \%[23.7-36.6]$ & 0.15 \\
aGVHD II-IV & $33.1 \%[28.6-37.6]$ & $30.5 \%[24.4-36.8]$ & 0.28 \\
cGVHD & $40.6 \%[35.3-45.9]$ & $26.5 \%[20.5-32.7]$ & 0.24 \\
ext cGVHD & $20.5 \%[16.2-25.1]$ & $10.7 \%[6.8-15.5]$ & 0.041 \\
\hline
\end{tabular}

Abbreviations: LFS, leukemia free survival; OS, overall survival; $R I$, relapse incidence; $N R M$, non-relapse mortality; GRFS, graft-versus-host-free-relapse free survival; $a$, acute; c, chronic; GVHD, graft versus host disease; ext., extensive
$84, p<0.01$ ), cytogenetic risk (HR 1.79, 95\% CI 1.25-2. $56, p<0.01$; HR 1.66, 95\% CI 1.14-2.40, $p<0.01$; HR 1. 69, 95\% CI 1.20-2.38, $p<0.01$ ), KPS (HR 0.85, 95\% CI 0. 76-0.96, $p=0.009$; HR 0.83, 95\% CI 0.74-0.94, $p=0.003$; HR $0.85,95 \%$ CI $0.76-0.94, p=0.003)$. Previous autograft was associated with lower OS (HR 1.39, 95\% CI 1. $00-1.93, p=0.04$ ) and LFS (HR 1.36, 95\% CI 1.00-1.86, $p=0.04)$. Importantly, OS was also influenced by incremental age (HR 1.16, 95\% CI 1.00-1.34, $p=0.04$ ).

\section{Discussion}

AML incidence increased with age and picked at the sixth-seventh decade of life; it is thus a disease of the elderly, and often at diagnosis, it is highly aggressive in this group of patients. Allo-SCT offers the best curative option with curative potential for these patients, despite the higher risk of complications related to transplant at advanced age as a result of frequent comorbidities and reduced compatible sibling donor availability at this age. Several studies demonstrated the feasibility of allo-SCT with the use of RIC in older population [6-8]. Recently, Rashidi et al. [36] reported 35 and $38 \%$ of 3 years LFS and OS, respectively, for elderly AML patients. With the development of new transplant techniques making alternative donors more realistic clinical option, when a MRD donor is not available, the question of which is the best alternative donor and the alternative donor hierarchy is still unanswered. MUD is known to have comparable outcomes to MRD allo-SCT [11-14], but it has the major disadvantage of rather long time for donor identification. On the other hand, Haplo-SCT is increasing worldwide, and it has the main advantage of providing a timely transplant thanks to rapid donor identification for almost all patients in need. 


\section{a}

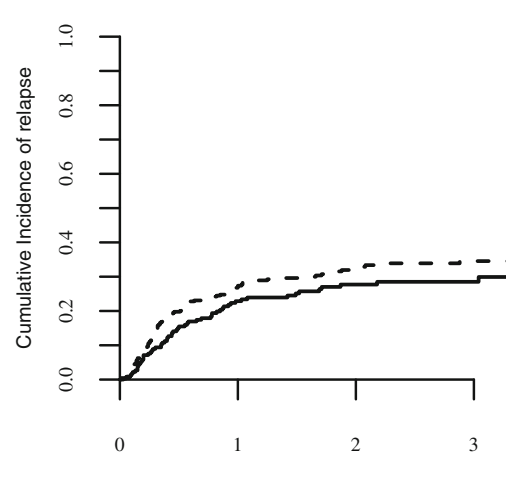

Time from transplant (years)
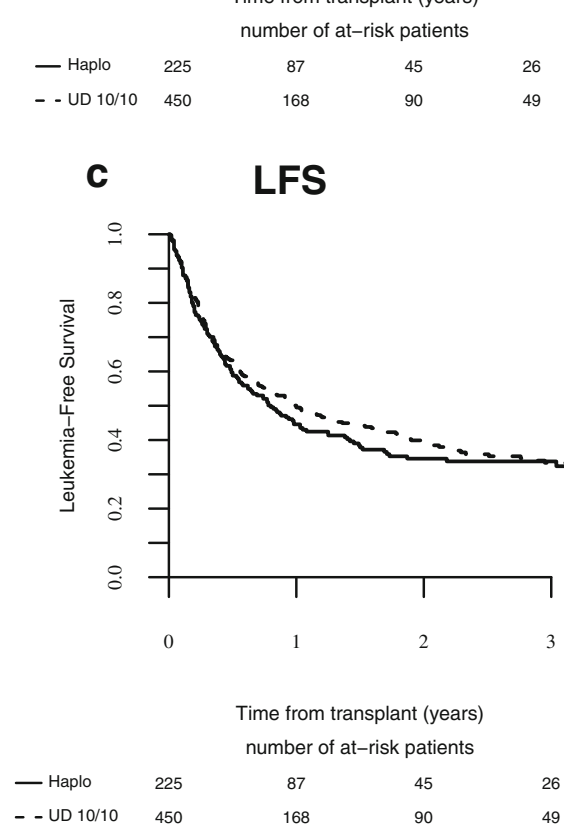

b NRM

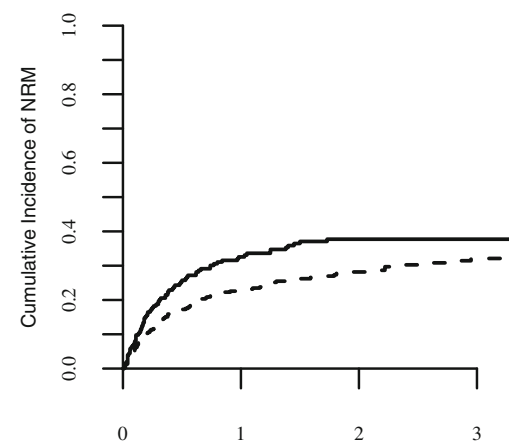

Time from transplant (years)

number of at-risk patients

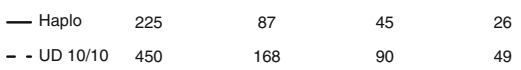

d os

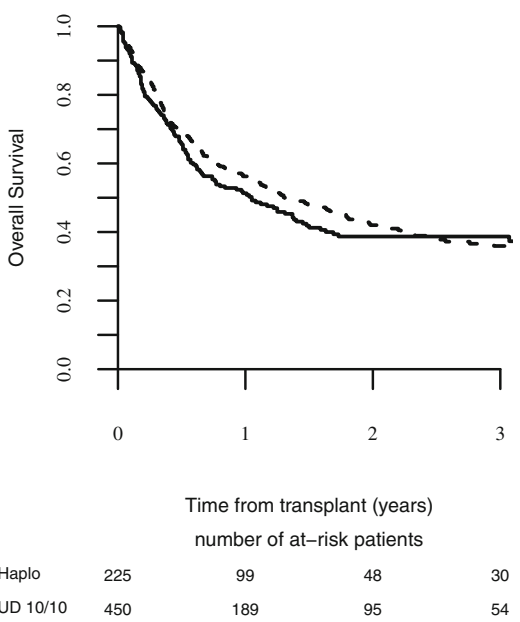

Fig. 2 a) Rl; b) NRM; c) LFS; d) OS after MUD and Haplo SCT in AML patients $\geq 60$ years after matched pair analysis

In the present study, we compared the results of alloSCT from Haplo donors or MUD 10/10 in a large number of AML patients $\geq 60$ years, in an attempt to better understand which is the best transplant choice in this setting.

Weisdorf et al. [37], evaluated the role of alternative donors in 740 older AML patients, but only 29 cases of Haplo-SCT were included in the study precluding a comparison for this type of donor.

Chen et al. using ATG based GVHD prophylaxis reported similar outcomes of myeloablative haploidentical SCT in patients aged $\geq 50$ years in comparison to younger adults (24). The same group also confirmed the efficacy of unmanipulated haplo-SCT compared to unrelated donors in young adult with hematological malignancies (25). The feasibility of Haplo-SCT with PT-Cy in the older patients was retrospectively analyzed by Kasamon et al. [26] in 271 patients with hematological malignancies (AML 24\%) receiving a RIC regimen with fludarabine, cyclophosphamide, and low dose of TBI. For AML patients aged > 60 years, the 3 years LFS, RI, and OS were 31, 60, and $38 \%$, respectively. These results are in line with ours, especially in terms of OS and LFS, despite the fact that in our analysis, being a registry-based study, we included different conditioning regimes and GVHD prophylaxis. Importantly, given the different type of GVHD prophylaxis in the haplo group, we performed a subgroup analysis according to the use or not of PT-Cy, comparing those two groups vs MUD. Our results confirm the decreased risk of extensive cGVHD for haplo recipients both in the group of PT-Cy and MUD, as well in the group of Haplo with an ATGbased GVHD prophylaxis (without PT-Cy) vs MUD.

In our population, age at transplant was an independent factor associated with reduced OS. Slade et al. [28] 
recently, retrospectively analyzed the impact of age on Haplo-SCT with PT-Cy and PBSC for AML or myelodysplastic syndrome (MDS), showing a detrimental effect of older age, in accordance with our results.

In a single center study, Blaise et al. [27] recently reported a comparison of outcome of patients older than 55 years receiving Haplo-SCT with PT-Cy, with those receiving MRD or MUD. Despite the low number of patients with AML reported (30\%), the results of this study showed that tolerability and efficacy of Haplo-SCT is equivalent to that of MRD and better than MUD. In our population, we did not find difference in OS, LFS, RI, and NRM, between transplantation from Haplo and MUD beside a difference in CGVHD. MUD is associated with higher incidence of cGVHD also when compared with MRD [38]. This could be explained by an effect of minor HLA mismatch and also by the increased use of PBSC in the MUD group, which is an important factor also reported by others [39]. Importantly, the application of PT-Cy as GVHD prophylaxis in the unrelated donor setting is attractive, and some encouraging results are reported [40]. Lastly, Ciurea et al. [29] recently analyzed 43 patients with AML/MDS (median age 61 years) who underwent a Haplo-SCT using PT- Cy. Factors that positively influenced LFS were intermediate/good risk cytogenetics in the first or second remission and younger donors $(<40$ years). These results are similar to ours, despite we included different platforms of Haplo-SCT in the current series. Importantly, in our study disease status was one of the main factors associated with outcomes. Using the propensity score analysis, we performed a separate analysis according to disease status, confirming the main results and the reduced risk of cGVHD for patients receiving haploidentical transplant in CR1.

We are aware that our study has some limitations related to its retrospective nature. Importantly, the conditioning regimen and the GVHD prophylaxis are heterogeneous, and thus, we were unable to define a specific drug combination in this particular population of patients.

In order to address the difference in number among the two groups, a propensity score analysis was performed which confirmed the same findings.

\section{Conclusions}

Given our results, we confirm that Haplo-SCT is a valid option in fit AML patients $\geq 60$ years, especially in patients in first complete remission, with intermediate/high cytogenetic risk. The rapid availability of Haplo donor and the possible use in further strategies of immunotherapy make this donor source very attractive for high-risk patients. A prospective clinical trial (ClinicalTrials.gov Identifier NCT02623309) comparing Haplo vs MUD in elderly patients without MRD is ongoing to address efficacy, safety, and the evaluation of quality of life. The results of this trial could help in clarifying the role of alternative donors in the elderly, to provide better cure in this high-risk population.

\begin{abstract}
Abbreviations
a: Acute; allo-SCT: Allogenic stem cell transplantation; ALWP: Acute Leukemia Working Party; AML: Acute myeloid leukemia; ATG: Anti-thymocyte globulins; BM: Bone marrow; Bu: Busulfan; C: Chronic; Cl: Cumulative incidence; EBMT: The European Society of Bone Marrow Transplantation; GRFS: Refined graft-versus-host-free, relapse-free survival; GVHD: Graft versus host disease; Haplo-SCT: Haploidentical transplant; KPS: Karnofsky performance status; LFS: Leukemia-free survival; MAC: Myeloablative conditioning; MDS: Myelodysplastic syndrome; MMUD: Mismatched unrelated donors; MRD: Matched related donor; MUD: Matched unrelated donors; NRM: Non-relapse mortality; OS: Overall survival; PBSC: Peripheral blood stem cell; PT-Cy: Post-Tx cyclophosphamide; RI: Relapse incidence; RIC: Reduced intensity conditioning; TBI: Total body irradiation; TCD: T-cell depletion; TX: Transplant; UCB: Umbilical cord blood
\end{abstract}

\section{Acknowledgements}

The authors thank Audrey Mailhol and Emmanuelle Polge for helping in the data collection.

\section{Consent for publications}

Not applicable

\section{Funding}

Not applicable

\section{Availability of data and materials}

The dataset supporting the conclusions of this article are available in the ALWP of EBMT in Paris, 184 rue Faubourg Saint Antoine.

\section{Authors' contributions}

$F G, M L, A R$, and $A N$ designed the study; NS and AR wrote the manuscript; $M L$ performed the statistical analysis; GE, DN, GS, MS, NK, HE, ME, MH, BG, JF, FC, and MM provided cases for the study. All authors edited and approved the manuscript.

\section{Ethics approval and consent to participate}

This study was approved by the ALWP of the EBMT institutional review board. The study was conducted in accordance with the Declaration of Helsinki and Good Clinical Practice guidelines. All patients or legal guardians provided written informed consent authorizing the use of their personal information for research purposes.

\section{Competing interests}

The authors declare that they have no competing interests.

\section{Publisher's Note}

Springer Nature remains neutral with regard to jurisdictional claims in published maps and institutional affiliations.

\section{Author details}

'Department of Hematology and Cell Therapy, Saint-Antoine Hospital, Paris, France. ${ }^{2}$ Section of Hematology, Department of Medicine, University of Perugia, Centro Ricerche Emato-Oncologiche (CREO), Perugia, Italy. ${ }^{3}$ ALWP office, Hôpital Saint-Antoine, Paris, France. ${ }^{4}$ Medical Clinic and Policlinic I, University Hospital Carl Gustav Carus, Technical University Dresden, Dresden, Germany. ${ }^{5}$ Department of Hematology and Oncology, University of Leipzig, Leipzig, Germany. ${ }^{6}$ Center for Blood Stem Cell and Bone Marrow Transplant, DKD Helios Clinic Wiesbaden, Wiesbaden, Germany. ${ }^{7}$ Department of Medicine A/Hematology and Oncology, University of Muenster, Muenster, Germany. ${ }^{8}$ Department of Stem Cell Transplantation, University Medical Center Hamburg-Eppendorf, Hamburg, Germany. ${ }^{9}$ Department of Internal Medicine II, University Hospital Wurzburg, Würzburg, Germany. ${ }^{10}$ Department of Hematology, Hemostasis, Oncology and Stem Cell Transplantation, Hannover Medical School, Hannover, Germany. ${ }^{11}$ Department I of Internal Medicine and Center of Integrated Oncology Cologne-Bonn, German CLL Study Group, University Hospital of Cologne, Cologne, Germany. 
${ }^{12}$ Department of Hematology and Oncology, Asklepios Klinik St. Georg Hamburg, Hamburg, Germany. ${ }^{13}$ Department of Medicine-Hematology, Oncology, University of Freiburg, Freiburg, Germany. ${ }^{14}$ Hematology and Bone Marrow Transplantation Unit, IRCCS San Raffaele Scientific Institute, Milan, Italy. ${ }^{15}$ Department of Pediatric Hematology and Oncology, IRCCS Bambino Gesù Children's Hospital, Piazza S Onofrio, 4, 00165 Rome, Italy. ${ }^{16}$ Department of Hematology and Bone Marrow Transplantation, Chaim Sheba Medical Center, Tel Hashomer, Israel.

Received: 16 February 2018 Accepted: 29 March 2018 Published online: 16 April 2018

\section{References}

1. Dohner H, Weisdorf DJ, Bloomfield CD. Acute myeloid leukemia. N Engl J Med. 2015;373:1136-52.

2. Roland BW, Estey EH. Management of older or unfit patients with acute myeloid leukemia. Leukemia. 2015;29:770-5.

3. Almeidaa AM, Ramosb F. Acute myeloid leukemia in the older adults. Leuk Res Rep. 2016;6:1-7.

4. Thein MS, Ershler WB, Jemal A, Yates JW, Baer MR. Outcome of older patients with acute myeloid leukemia: an analysis of SEER data over 3 decades. Cancer. 2013;119:2720-7.

5. Wallen $\mathrm{H}$, Gooley TA, Deeg HJ, et al. Ablative allogeneic hematopoietic cell transplantation in adults 60 years of age and older. J Clin Oncol. 2005;23:3439-46.

6. Ringden $\mathrm{O}$, Labopin $\mathrm{M}$, Ehninger $\mathrm{G}$, et al. Reduced intensity conditioning compared with myeloablative conditioning using unrelated donor transplants in patients with acute myeloid leukemia. J Clin Oncol. 2009;27:4570-7.

7. McClune BL, Weisdorf DJ, Pedersen TL, et al. Effect of age on outcome of reduced-intensity hematopoietic cell transplantation for older patients with acute myeloid leukemia in first complete remission or with myelodysplastic syndrome. J Clin Oncol. 2010;28:1878-188.

8. Estey $E$, de Lima $M$, Tibes $R$, et al. Prospective feasibility analysis of reduced intensity conditioning regimens for hematopoietic stem cell transplantation in elderly patients with acute myeloid leukemia and high risk myelodisplastic syndrome. Blood. 2007;109:1395-400.

9. Muffly L, Pasquini MC, Martens M, et al. Increasing use of allogeneic hematopoietic cell transplantation in patients aged 70 years and older in the United States. Blood. 2017;130:1156-64.

10. Tiercy JM. How to select the best available related or unrelated donor of hematopoietic stem cells? Haematologica. 2016;101:680-7.

11. Yakoub-Agha I, Mesnil F, Kuentz M, et al. Allogeneic marrow stem-cell transplantation from human leukocyte antigen-identical siblings versus human leukocyte antigen-allelic-matched unrelated donors (10/10) in patients with standard-risk hematologic malignancy: a prospective study from the French Society of Bone Marrow Transplantation and Cell Therapy. J Clin Oncol. 2006;24:5695-702.

12. Walter RB, Pagel JM, Gooley TA, et al. Comparison of matched unrelated and matched related donor myeloablative hematopoietic cell transplantation for adults with acute myeloid leukemia in first remission. Leukemia. 2010;24:1276-82.

13. Ruggeri A, Battipaglia G, Labopin M, et al. Unrelated donor versus matched sibling donor in adults with acute myeloid leukemia in first relapse: an ALWP-EBMT study. J Hematol Oncol. 2016;9:89.

14. Brissot $E$, Labopin $M$, Stelljes $M$, et al. Comparison of matched sibling donors versus unrelated donors in allogeneic stem cell transplantation for primary refractory acute myeloid leukemia: a study on behalf of the Acute Leukemia Working Party of the EBMT. J Hematol Oncol. 2017;10:130.

15. Schetelig J, Bornhauser M, Schmid C, et al. Matched unrelated or matched sibling donors result in comparable survival after allogeneic stem-cell transplantation in elderly patients with acute myeloid leukemia: a report from the cooperative German Transplant Study Group. J Clin Oncol. 2008; 26:5183-91.

16. Gragert L, Eapen M, Williams E, et al. HLA match likelihoods for hematopoietic stem cell grafts in the US registry. N Engl J Med. 2014;371:339-48.

17. Passweg JR, Baldomero H, Bader P, et al. Use of haploidentical stem cell transplantation continues to increase: the 2015 European Society for Blood and Marrow Transplant activity survey report. Bone Marrow Transplant. 2017;52:811-7.

18. Kanakry CG, Fuchs EJ, Luznik L. Modern approaches to HLA-haploidentical blood or marrow transplantation. Nat Rev Clin Oncol. 2016;13:10-24.

19. Luznik L, O'Donnel PV, Fuchs EJ. Post transplantation cyclophosphamide for tolerance induction in HLA haploidentical BMT. Seminar Oncol. 2012;39:683-93.
20. Chang YJ, Huang XJ. Haploidentical stem cell transplantation: antithymocyte globulin-based experience. Semin Hematol. 2016;53:82-9.

21. Castagna L, Blaise D, Furst S. Allogeneic hematopoietic stem cell transplantation in elderly. Bull Cancer. 2011;98:915-25.

22. Chevallier P, Szydlo RM, Blaise D, et al. Reduced-intensity conditioning before allogeneic hematopoietic stem cell transplantation in patients over 60 years: a report from the SFGM-TC. Biol Blood Marrow Transplant. 2012;18:289-94.

23. Blaise $D$, Devillier $R$, Lecoroller-Sorriano $A G$, et al. Low non-relapse mortality and long-term preserved quality of life in older patients undergoing matched related donor allogeneic stem cell transplantation: a prospective multicenter phase II trial. Haematologica. 2015;100:269-74.

24. Chen $Y$, Wang $Y, X u L P$, et al. Haploidentical stem cell transplantation in patients aged $50 \mathrm{yr}$ and older with leukemia: similar outcomes compared to younger adults. Clin Transpl. 2015;29:523-30.

25. Xiao-Jun $H$, Lan-Ping X, Kai-Yan L, et al. Partially matched related donor transplantation can achieve outcomes comparable to unrelated donor transplantation for patients with hematologic malignancies. Clin Cancer Res. 2009;15:4777-83.

26. Kasamon YL, Bolaños-Meade J, Prince GT, et al. Outcomes of nonmyeloablative HLA-haploidentical blood or marrow transplantation with high-dose post-transplantation cyclophosphamide in older adults. J Clin Oncol. 2015:33:3152-61.

27. Blaise $D$, Fürst $S$, Crocchiolo R, et al. Haploidentical T cell-replete transplantation with post-transplantation cyclophosphamide for patients in or above the sixth decade of age compared with allogeneic hematopoietic stem cell transplantation from an human leukocyte antigen-matched related or unrelated donor. Biol Blood Marrow Transplant. 2016;22:119-24.

28. Slade M, DiPersio JF, Westervelt P, Vij R, Schroeder MA, Romee R. Haploidentical hematopoietic cell transplant with post-transplant cyclophosphamide and peripheral blood stem cell grafts in older adults with acute myeloid leukemia or myelodysplastic syndrome. Biol Blood Marrow Transplant. 2017;23:1736-43.

29. Ciurea SO, Shah MV, Saliba RM, et al. Haploidentical transplantation for older patients with acute myeloid leukemia and myelodysplastic syndrome. Biol Blood Marrow Transplant. 2017; https://doi.org/10.1016/j.bbmt.2017.09.005

30. Bacigalupo A, Ballen K, Rizzo D, et al. Defining the intensity of conditioning regimens: working definitions. Biol Blood Marrow Transplant. 2009;15:1628-33.

31. Dohner H, Estey EH, Amadori S, et al. Diagnosis and management of acute myeloid leukemia in adults: recommendations from an international expert panel, on behalf of the European LeukemiaNet. Blood. 2010;115:453-74.

32. Ruggeri A, Labopin M, Ciceri F, et al. Definition of GVHD-free, relapse-free survival for registry-based studies: an ALWP-EBMT analysis on patients with AML in remission. Bone Marrow Transplant. 2016;51:610-1.

33. Przepiorka D, Weisdorf D, Martin P, et al. 1994 Consensus conference on aGvHD grading. Bone Marrow Transplant. 1995;15:825-8.

34. Lee SJ, Vogelsang G, Flowers ME. Chronic graft versus host disease. Biol Blood Marrow Transplant. 2003;9:215-33. 28

35. McCaffrey D, Griffin BA, Almirall D, Slaughter ME, Ramchand R, Burgette LF. A tutorial on propensity score estimation for multiple treatments using generalized boosted models. Stat Med. 2013;32:3388-414.

36. Rashidi A, Ebadi M, Colditz GA, DiPersio JF. Outcomes of allogeneic stem cell transplantation in elderly patients with acute myeloid leukemia: a systematic review and meta-analysis. Biol Blood Marrow Transplant. 2016;22:651-7.

37. Weisdorf D, Eapen M, Ruggeri A, et al. Alternative donor transplantation for older patients with acute myeloid leukemia in first complete remission: a Center for International Blood and Marrow. Transplant Research-Eurocord analysis. Biol Blood Marrow Transplant. 2014;20:816-22.

38. Saber W, Opie S, Rizzo JD, Zhang MJ, Horowitz MM, Schriber J. Outcomes after matched unrelated donor versus identical sibling hematopoietic cell transplantation in adults with acute myelogenous leukemia. Blood. 2012;119:3908-16.

39. Anasetti C, Logan BR, Lee SJ, et al. Peripheral-blood stem cells versus bone marrow from unrelated donors. N Engl J Med. 2012;367:1487-96.

40. Kanakry CG, O'Donnell PV, Furlong T, et al. Multi-institutional study of posttransplantation cyclophosphamide as single-agent graft-versus-host disease prophylaxis after allogeneic bone marrow transplantation using myeloablative busulfan and fludarabine conditioning. J Clin Oncol. 2014;32:3497-505. 